\title{
Minimising radiation dose using pulsed digital fluoroscopy in the investigation of children
}

\section{Savvas Andronikou MB BCh, FC Rad (SA), FRCR (Lond)}

\section{Marisa Mezzabotta MB BCh \\ Department of Paediatric Radiology Red Cross Children's Hospital School of Child and Adolescent Health University of Cape Town}

recommended protocol for performing a micturating cystourethrogram and a barium swallow/meal in children.

\section{Introduction}

In children, fluoroscopy is most frequently used in the investigation of urinary tract infection ${ }^{1}$ (micturating cystourethrography (MCUG)) and in screening those children suspected of gastro-oesophageal reflux (barium swallow/meal). Paediatric radiology is especially concerned with minimising radiation dose because of the higher risk of radiogenic cancers in children due to medical irradiation. ${ }^{1,2}$ The goal of fluoroscopic studies is to produce interpretable images at the lowest possible radiation exposure and dosage to the patient. . $^{-4}$

\section{Aim}

To describe the advantages of pulsed digital fluoroscopy and to present our recommendations for its use in imaging urinary tract infection and gastro-oesophageal reflux in children with respect to minimising radiation dose.

\section{Discussion}

Most modern fluoroscopic systems use digital technology (as opposed to analogue). These systems digitise the image offering the advantages of image enhancement (post processing) after the image has been stored, and result in a reduction of radiation dose. ${ }^{5}$ Images can be stored as 'picture-grabs', 'cine-loops' or 'spot films'. A 'picture-grab' is a stored image of a single fluoroscopic frame at fluoroscopic doses. Diagnostic information is, however, not lost in the imaging of UTI in children. ${ }^{4}$ A cineloop' is a stored series of flouoroscopic frames that can be replayed in rapid sequence, much like a video clip, once again at fluoroscopic doses. A spot film is a radiographic film/screen quality digital image. Film quality is significantly improved compared with 'picture-grabs' or 'cine-loops', but there is added radiation exposure. ${ }^{6}$

Modern fluoroscopy systems also offer two modes of fluoroscopy. Either the X-ray beam is continuous or the $\mathrm{X}$-ray beam is emitted for a few strobe-like X-ray pulses each second.

Pulsing the X-ray beam can reduce radiation exposure to both patients and radiology staff. ${ }^{7.8}$ Dose is reduced by simply decreasing the fraction of time that the $\mathrm{X}$-ray beam is emitted. A range of pulsing is offered, from 3 pulses per second (pps) to $30 \mathrm{pps}$, while the choice of continuous fluoroscopy is also available. When using a 7.5 pps rate, the radiation exposure from fluoroscopy is approximately $75 \%$ lower than that from conventional fluoroscopy. ${ }^{8}$ It is therefore up to the operator to choose whether to use pulsed fluoroscopy and what rate of pulsing to use in each individual case. 
Other than radiation saving, pulsed fluoroscopy offers the advantage of better temporal resolution of moving objects. ${ }^{2}$ Each image of the moving object is sharper than with continuous fluoroscopy because the object is relatively still during each short pulse of X-rays. ${ }^{2,6}$ The disadvantage of pulsed fluoroscopy is that the fewer frames result in the perception of increased noise because the eye averages fewer independent frames, ${ }^{8}$ as well as image animation (jumpiness). ${ }^{3.8}$ The lower the pulse rate, the higher the perceived image noise. ${ }^{8}$ The percentage of noise at $7.5 \mathrm{pps}$ is twice that at $30 \mathrm{pps}$. Actual image quality is perceived to be noisiest but acceptable at 7.5 pps. $^{8}$

Most paediatric fluoroscopic investigations are performed as single contrast techniques with the aim of answering a specific question. In the investigation of UTI, the primary objectives are to rule out posterior urethral valves (in males) and to exclude vesico-ureteric reflux. During a barium swallow/meal the radiologist aims to exclude impressions on the oesophagus, malrotation and gastrooesophageal reflux. The radiologist and clinician must then accept that noisier images can yield the same diagnostic information as conventional images even though quality may be decreased. ${ }^{4}$ This is a psychological barrier ${ }^{3}$ and radiologist acceptance of low-radiation images is a vital factor in pulsed fluoroscopy. ${ }^{2}$

The following paragraphs and images describe our recommended method of performing micturating cysto-urethrography and barium swallow/meal at the Red Cross Children's Hospital. The protocols include the ideal choice of pulsing, the required views and the recommended method of image storage ('grab' versus 'spot film'). The aim is to store adequate diagnostic information at the lowest radiation dose possible.

Remember only to screen intermittently and to collimate tightly! Collimation can be achieved without radiation by using the positioning light. We begin fluoroscopy using 7.5 pps and this is changed when necessary.

\section{MCUG}

1. After catheterising the patient maintain the right side down oblique position throughout the procedure. No preliminary film is necessary. ${ }^{9}$ Begin to fill the bladder and 'grab' the bladder-filling image. This is intended to confirm the position of the catheter and assess for early vesico-ureteric reflux (Fig. 1).

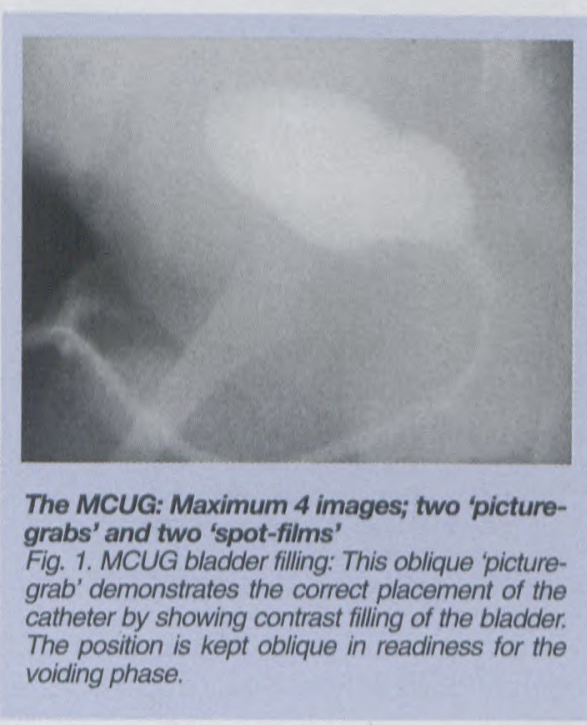

\section{Continue intermittent fluo-} roscopy until the patient begins to void and perform a 'spot-film' while the patient voids with or without the catheter in situ (valves can be diagnosed with the catheter in) (Fig. 2). ${ }^{10}$ Ensure that the back of the bladder is visible so that any vesico-ureteric
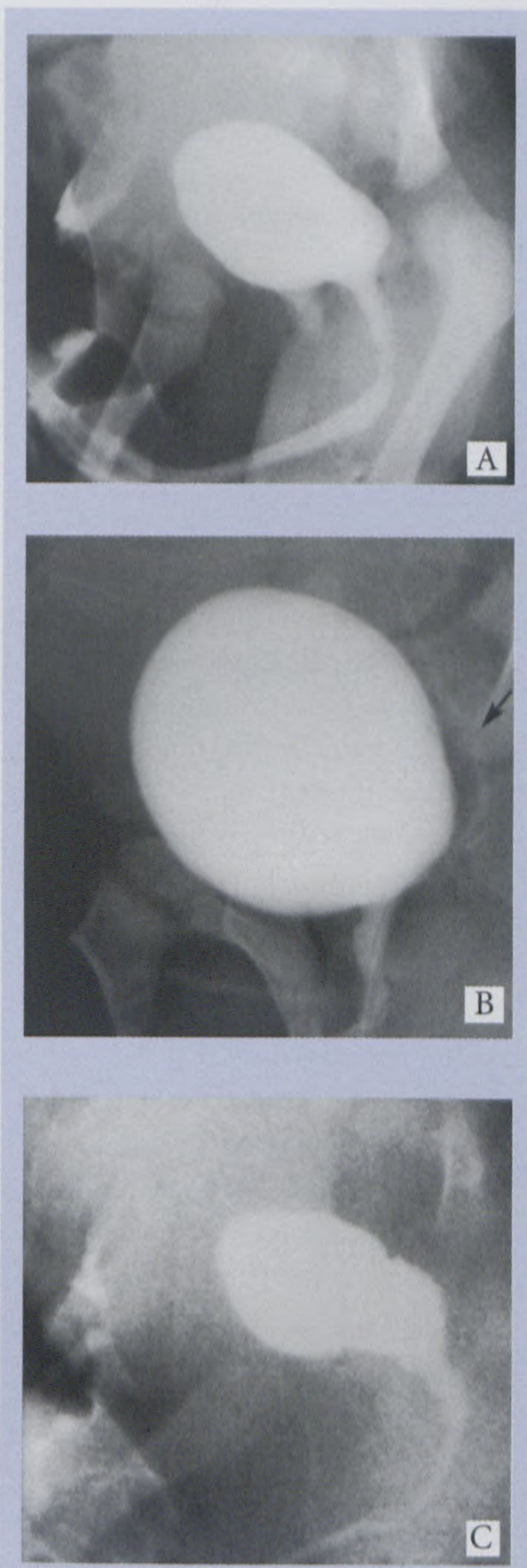

Figs $2 a, b$ and c. Voiding image: $2 a$. 'Spot-film' in the oblique position demonstrates a normal urethra in a male. $2 b$. By including the posterior portion of the bladder, this 'spot-film' in the oblique position demonstrates grade 1 vesicoureteric reflux during the voiding phase (arrow). Performing the contralateral oblique image is not recommended as the voiding phase may be missed, which is important for documenting urethral anatomy and is also the time when reflux is most likely to occur. Minimal contralateral reflux may be missed, however this would not be considered significant.

2c. Voiding phase of an MCUG recorded using a 'picture-grab' is of lower quality but is adequate for diagnostic information. 


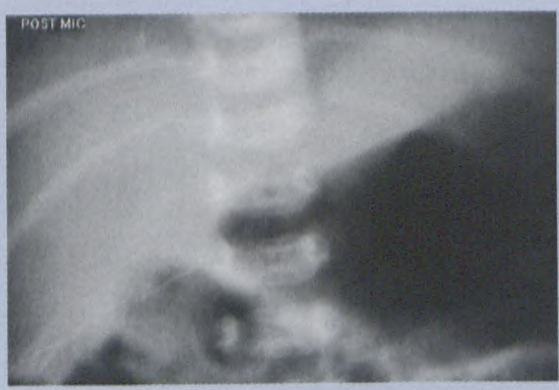

Fig. 3. Renal area: A 'spot-film' of the renal area is recommended after the voiding film to exclude contrast within the calyces. A 'picture-grab' may not provide enough detail to distinguish contrast in the calyces from other normal structures.

reflux will be recorded and ensure that the tip of the penis is shown to exclude diverticula and anterior urethral valves. Optionally this could be a 'picture-grab' image. ${ }^{4,8}$

3. When voiding is complete, perform a tightly collimated renal 'spotfilm'. Detail is required for this image to identify contrast in the calyces and therefore a 'spot-film' is recommended, but not obligatory (Fig. 3).

4. Screen over the bladder, and if residual urine is present in the bladder, record this using a 'picture-grab' in the AP position. Avoid unnecessary spot films of the pelvic area for this purpose.

\section{Barium swallow/meal}

1. The child is placed in the left true lateral position and is fed barium via bottle, syringe or straw. The fullcolumn oesophagus is viewed in the true lateral position and a full-length image is stored as a 'picture-grab', which is adequate for evaluating the gastrointestinal tract (Fig. 4). ${ }^{8}$ This allows exclusion of vascular impressions on the oesophagus.

2. The patient is then turned to the right true lateral position with tight collimation of the stomach and duodenal region. An image of early duo-

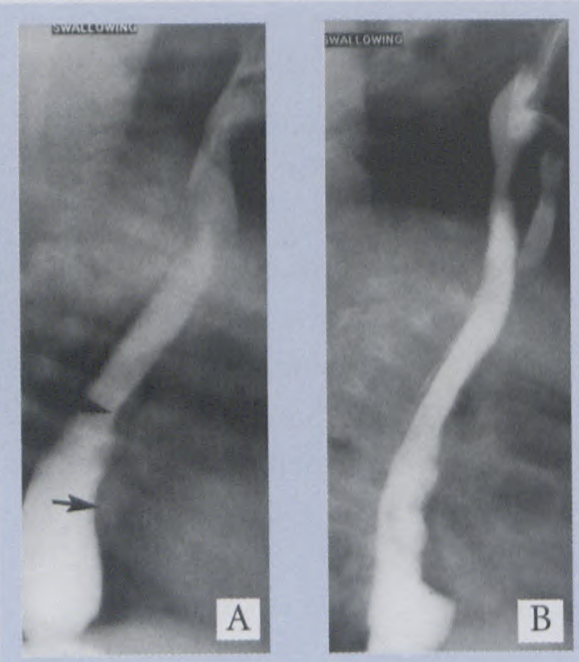

The Barium Swallow/Meal: Maximum 4 images; all 'picture-grabs'

Figs $4 a$ and $b$. 'Picture-grab' of the lateral oesophagus: $4 a$. True lateral 'picture-grab' of the oesophagus with a full column of barium demonstrates the normal impressions on the anterior aspect (arrows). 4b. Lateral 'picture-grab' demonstrates the aspirated contrast in the trachea adequately in this child with incoordinated swallowing.

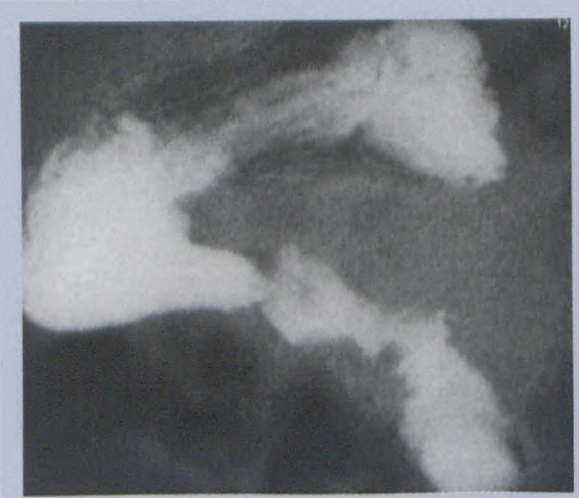

Fig. 5. Lateral stomach and duodenum: A picturegrab' of the stomach and duodenum in the right side down lateral position demonstrates the normal retroperitoneal course of the duodenum for exclusion of malrotation.

denal filling is 'grabbed' (Fig. 5). This true lateral image gives information that the duodenum is oriented posteriorly, to exclude malrotation.

3. Rapidly turn the patient to the AP position and collimate tightly to the stomach and duodenal C-loop. Include the base of the heart and make sure the spine is truly AP. A 'picture-grab' of a full C-loop is then

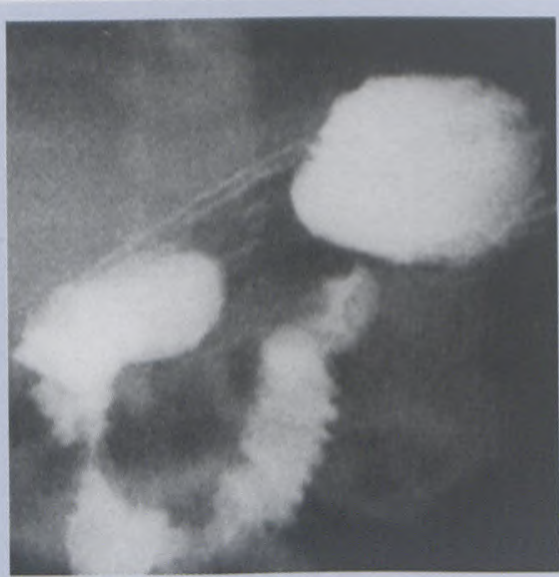

Fig. 6. Duodenal C-loop: A 'picture-grab' of the contrast-filled duodenal C-loop in the AP position is mandatory for exclusion of malrotation in every patient undergoing a barium swallow/meal, whatever the indication.

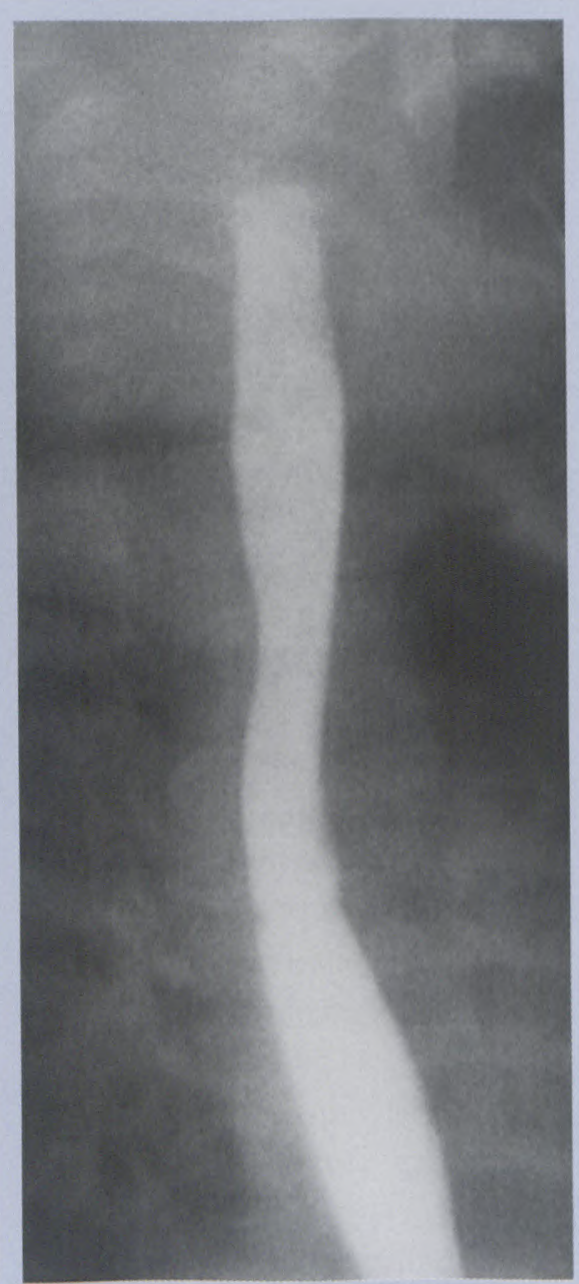

Fig. 7. Gastro-oesophageal reflux: If gastrooesophageal reflux is present, a 'picture-grab' is adequate for recording this, as in this patient with contrast refluxing to the level of the hypopharynx. 
stored (Fig. 6). Try to avoid contrast beyond the 4 th part of the duodenum as this compromises your ability to confirm its position and therefore your ability to exclude malrotation.

4. The patient may now be given more oral contrast in the left lateral position to fill the stomach. In the AP position, the patient is screened intermittently for gastro-oesophageal reflux over 3 minutes (NOT $3 \mathrm{MIN}$ UTES OF SCREENING). If reflux is seen, then the image is stored as a 'picture-grab' (Fig. 7).

\section{Reminders}

Remember that fluoroscopic doses (when used intermittently) ${ }^{7}$ are far lower than fluoroscopy plus 'spotfilm' doses and that 'picture-grabs' are stored images of fluoroscopy. Reserve 'spot-films' for those situations when detail is required. If a pathologic abnormality is identified, further views may be warranted at higher fluoroscopic pulse rates, with the use of continuous fluoroscopy or with the use of 'spot-films', available at the touch of a button. Also keep in mind that tight collimation reduces radiation and that a good paediatric radiol- ogist keeps his foot on the fluoroscopy pedal for only a few seconds at a time.

\section{Conclusion}

Digital systems have allowed us the opportunity to improve an image after it has been stored. The need for repeat images has been reduced using this. With cine loop technology, the radiologist also has the advantage of screening a full swallow or bladder void and then selecting the 'picturegrab' image from the series in retrospect. If we as radiologists get through the psychological barrier of 'ugly' images, we can certainly reduce the dose to our patients. Currently, all male children with UTI undergo MCUG, but the trend with females is to perform lower dose scintigraphic studies. Monitoring $\mathrm{pH}$ and scintigraphic studies are probably more successful alternatives for investigating gastro-oesophageal reflux in children but offer little anatomic information. Fluoroscopy in children is still being performed extensively throughout the world with a relatively low pick-up of pathology. It is our duty to use our equipment and modify our techniques to reduce radiation expo- sure. Pulsed fluoroscopy is considered a requisite for optimal paediatric fluoroscopy. ${ }^{2}$

\section{References}

1. Wilkinson AG, Mc Cafferty G. Pressure-assisted micturating cystourethrography: Reduction in duration of procedure and fluoroscopy time. Pediatr Radiol 2002; 32: 783-786.

2. Brown PH, Thomas RD, Silbeberg PJ, Johnson LM. Optimization of a fluoroscope to reduce radiation exposure in pediatric imaging. Pediatr Radiol 2000; 30: 229-235.

3. Wessenberg RL, Amundson GM. Fluoroscopy in children: Low exposure technology. Radiology 1984; 153: 243-247.

4. Bazopoulos EV, Prassopoulos PK, Damilakis JE, Raissaki MT, Megremis SD, Gourtsoyiannis NC. A comparison between digital fluoroscopic hard copies and $105 \mathrm{~mm}$ spot films in evaluating vesicoureteric reflux in children. Pediatr Radiol 1998; 28: 162-166.

5. Gleijins J, Broerse J, Chandie Shaw MP, et al. A comparison of patient dose for examinations of the upper gastrointestinal tract at 11 conventional and digital X-ray units in the Netherlands. Br J Radiol 1998; 71: 745-753.

6. Brown PH, Silbeberg PJ, Thomas RD, Strife JL, Towbin RB. A multihospital survey of radiation exposure and image quality in pediatric fluoroscopy. Pediatr Radiol 2000; 30: 236-242.

7. Mahesh M. Fluoroscopy: Patient radiation exposure issues. Radiographics 2001; 21: 1033 1045.

8. Hernandez RJ, Goodsitt MM. Reduction of radiation dose in pediatric patients using pulsed fluoroscopy. AJR 1996; 167: 1247-1253.

9. Ditchfield MR, De Campo JF. Is the preliminary film necessary prior to micturating cystourethrography in children? Abdom Imaging 1993; 18(2): 191-192.

10. Ditchfield MR, Grattan-Smith JD, De Campo JF, Hutson JM. VCU in boys: Does the presence of the catheter obscure the diagnosis of posterior urethral valves? AJR 1995; 164: 1233-1235. 